\title{
Targeted therapy using nanotechnology: focus on cancer
}

\author{
This article was published in the following Dove Press journal: \\ International Journal of Nanomedicine \\ 15 January 2014 \\ Number of times this article has been viewed
}

\author{
Vanna Sanna \\ Nicolino Pala \\ Mario Sechi \\ Department of Chemistry \\ and Pharmacy, Laboratory \\ of Nanomedicine, University \\ of Sassari, Sassari, Italy
}

Correspondence: Mario Sechi Department of Chemistry and Pharmacy, Laboratory of Nanomedicine, University of Sassari, Via Vienna 2, 07100, Sassari, Italy

Tel +390 79228753

Fax +390 79229559

Email mario.sechi@uniss.it
Abstract: Recent advances in nanotechnology and biotechnology have contributed to the development of engineered nanoscale materials as innovative prototypes to be used for biomedical applications and optimized therapy. Due to their unique features, including a large surface area, structural properties, and a long circulation time in blood compared with small molecules, a plethora of nanomaterials has been developed, with the potential to revolutionize the diagnosis and treatment of several diseases, in particular by improving the sensitivity and recognition ability of imaging contrast agents and by selectively directing bioactive agents to biological targets. Focusing on cancer, promising nanoprototypes have been designed to overcome the lack of specificity of conventional chemotherapeutic agents, as well as for early detection of precancerous and malignant lesions. However, several obstacles, including difficulty in achieving the optimal combination of physicochemical parameters for tumor targeting, evading particle clearance mechanisms, and controlling drug release, prevent the translation of nanomedicines into therapy. In spite of this, recent efforts have been focused on developing functionalized nanoparticles for delivery of therapeutic agents to specific molecular targets overexpressed on different cancer cells. In particular, the combination of targeted and controlled-release polymer nanotechnologies has resulted in a new programmable nanotherapeutic formulation of docetaxel, namely BIND-014, which recently entered Phase II clinical testing for patients with solid tumors. BIND-014 has been developed to overcome the limitations facing delivery of nanoparticles to many neoplasms, and represents a validated example of targeted nanosystems with the optimal biophysicochemical properties needed for successful tumor eradication.

Keywords: cancer, nanomedicine, targeted nanoparticles, BIND-014

\section{Introduction}

The application of nanotechnology in medicine is providing significant opportunities and new perspectives for novel and effective treatments in many disorders, with great potential in health care. Nanomedicine can be defined as the design and development of therapeutics and/or diagnostic agents in the nanoscale range (with diameters ranging from $1 \mathrm{~nm}$ to $1,000 \mathrm{~nm}$ ), with the possibility, by moving within biological systems, to transport and deliver a variety of biomedical entities for the treatment, prevention, and diagnosis of many diseases (Figure 1). ${ }^{1-5}$

Biological transport processes, from external barriers (skin and mucosa), en route compartments (blood and extracellular matrix), and cellular membranes, to destinations at the cellular and subcellular levels are affected by the physical features of nanocarriers, including their size, shape, surface charge, and intrinsic chemical properties, as well as the incorporation of active ligands for recognition of biological receptors. ${ }^{6-10}$ 


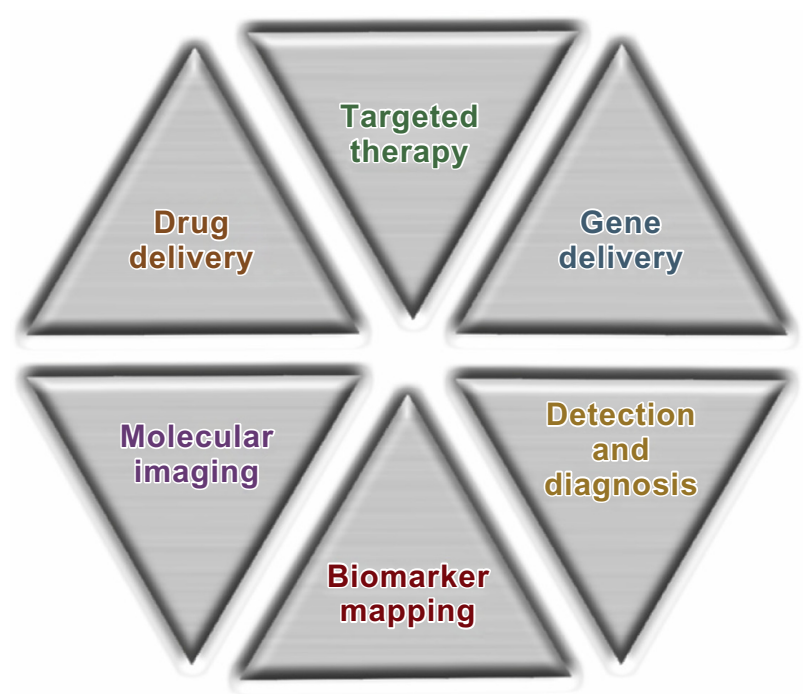

Figure I Biomedical applications of nanotherapeutics.

Due to their unique characteristics, including large surface area, structural properties, and long circulation time in blood compared with small molecules, nanoparticles have emerged as attractive candidates for optimized therapy through personalized medicine. ${ }^{11,12}$ Potential advantages of engineered therapeutic nanoparticles are the ability to: revert unfavorable physicochemical properties of bioactive molecules to desirable biopharmacologic profiles; improve delivery of therapeutics across biological barriers and compartments; control release of bioactive agents; enhance therapeutic efficacy by selective delivery of therapeutics to biological targets; and perform theranostic functions by combining multimodal imaging and simultaneous diagnosis and therapy into multifunctional nanoplatforms. ${ }^{13-18}$

Over the last few decades, many original tools have been developed based on various components from metals to proteins, including carbon, silica oxides, metal oxides, nanocrystals, lipids, polymers, dendrimers, and quantum dots, as well as newly developed materials. ${ }^{1,2,4,9,19-23}$ For example, carbon nanomaterials with a carbon cage (eg, fullerenes, nanodiamonds) and graphene structures (eg, carbon nanotubes, nanohorns) have been explored as carriers for drug delivery and other biomedical applications, ${ }^{24-27}$ due to their high variability, chemical stability, and unique characteristics, such as highly tailorable surface chemistry and high carrier capacity, and the feasibility of incorporating a variety of molecules as anticancer therapeutics. However, to enable drug delivery platforms, the major emphasis should lie in investigation of the mechanism, thermodynamics, and kinetics of adsorption/desorption equilibria for putative drugs on/from carbon nanomaterials with differing purity, surface chemistry, and agglomeration state under different conditions. Moreover, a detailed understanding of the pharmacologic and toxicologic properties of these nanomaterials, and a balanced and detailed evaluation of their risks and benefits to human health, are expected before their translation into clinical use. New perspectives using innovative nanomaterials for cancer treatment have been offered by a multifunctional platform based on gold nanoparticles, with the possibility of combining imaging and therapy, and also implementing multiple receptor targeting. ${ }^{28-32}$ Gold nanospheres, nanorods, nanoshells, and nanocages, for example, are currently being investigated for in vivo imaging, cancer therapy, and drug delivery. In particular, most studies of gold nanoparticle-based cancer therapy have involved a photothermal approach by near-infrared region laser exposure for the destruction of cancer cells or tumor tissue, which might have great potential in the clinical setting. ${ }^{29-31}$ Thus, laser-exposed gold nanoparticles could act as therapeutic agents by themselves without the need for coconjugated drugs. In addition, gold nanoparticles have been used in exploratory drug delivery applications by: partitioning and diffusion-driven release of hydrophobic drug molecules; loading therapeutics by surface complexation; anchoring drugs directly to particle surfaces; loading by layer-by-layer assembly; and loading drugs inside nanoparticles. ${ }^{32}$

As far as drug delivery is concerned, the most important nanoparticle platforms are liposomes, polymer conjugates, polymeric micelles, dendrimers, nanoshells, and protein and nucleic acid-based nanoparticles. ${ }^{1-3,6,7}$ Among these, the two prominent types of nanoparticles, ie, liposomes and polymerbased nanoformulations, constitute the majority of the nanoparticle therapeutics available for clinical use (Figure 2). ${ }^{7,10,15,33-40}$

Although these materials are likely to provide a high degree of biocompatibility, their utilization in biomedicine requires controlled interactions with biomacromolecules. To translate developed nanomedicines into clinical practice successfully, several issues, including a favorable blood halflife and physiologic behavior with minimal off-target effects, effective clearance from the human organism, and minimal or no toxicity to healthy tissues in living organisms, should be taken into consideration. ${ }^{10,41}$ More specifically, hydrodynamic diameter, surface charge, and hydrophobic/hydrophilic balance are important physicochemical properties that can affect the in vivo biodistribution and clearance of administered nanoparticles. ${ }^{10,42}$ For example, optimal nanoparticle surface modification using appropriate biocompatible and biodegradable polymers, such as polyethylene glycol (PEG), constitutes a suitable strategy to reduce opsonization and uptake processes in the reticuloendothelial system, also facilitating efficient 

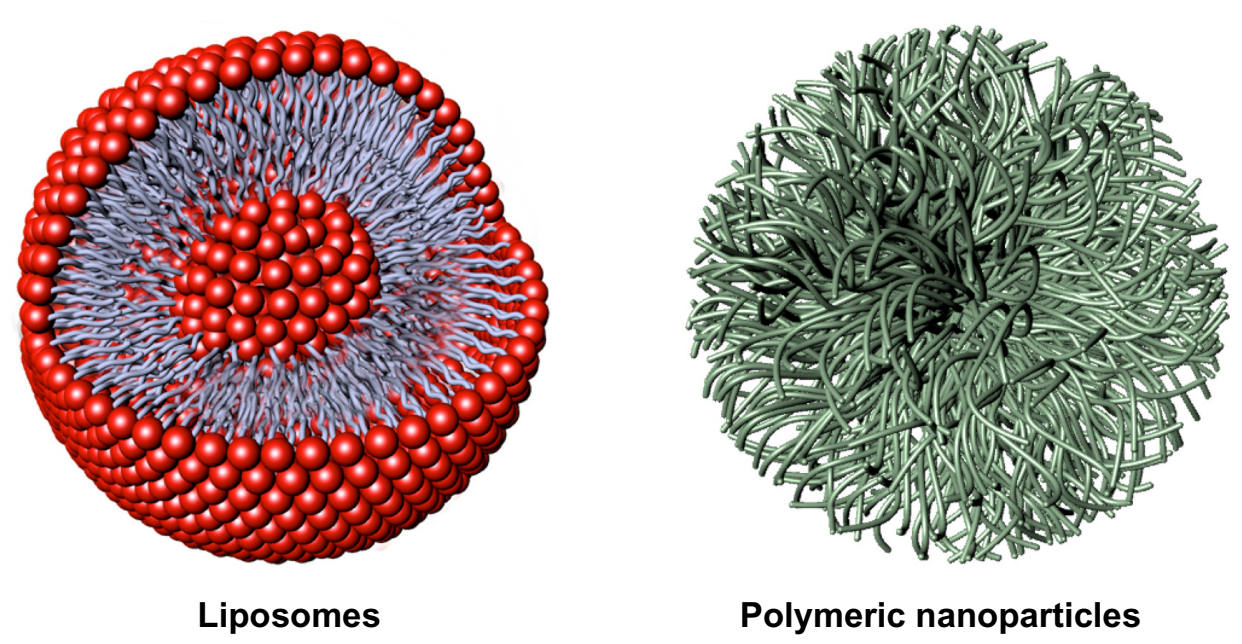

Polymeric nanoparticles

Figure 2 Representative nanocarriers for drug delivery, ie, liposomes (left) and polymeric nanoparticles (right). Liposomes are self-assembling vesicles with a bilayered membrane structure containing amphiphilic molecules (phospholipids) and hydrophobic and hydrophilic groups that self-assemble in water. Polymeric nanoparticles are biocompatible and biodegradable polymeric nanoformulations in which drugs are dissolved, entrapped, or conjugated to the surface of the nanoparticles.

clearance of nanoparticles and/or their metabolites from the body. ${ }^{36,43-47}$ Therefore, to achieve a favorable blood half-life and biodistribution with minimal recognition and elimination by the reticuloendothelial system, great effort should be devoted to optimizing the physicochemical profile of nanoparticles.

This review highlights recent advances in the development of targeted controlled-release nanoparticles for antitumor chemotherapy, starting from the potential impact of nanotechnology in medicine, through to the clinical development of emerging passive and active targeting nanoparticles for effective controlled drug delivery. Moreover, the evolution of BIND-014, the first clinically tested targeted nanomedicine for management of cancer, as well as its pharmacobiologic properties and potential within the chemotherapeutic arena, are herein detailed.

\section{Emerging and current nanomedicines and targeting methods: focus on cancer}

The incidence of cancer has been increasing in recent decades, and eradication of the major types of the disease remains an elusive clinical goal, largely due to the heterogeneous and idiosyncratic nature of individual cancers, and the inability to target therapeutics to neoplastic areas without damaging normal tissues. ${ }^{48,49}$ Most of the antitumor agents currently administered by validated therapeutic protocols are systemically distributed without preferential localization to cancer tissue. This widespread biodistribution of chemotherapeutics results in both anticancer effects and off-target adverse effects. Thus far, a few nanotherapeutic (and diagnostic) materials have been approved by the US Food and Drug Administration (FDA) for clinical use, although more are currently in various stages of preclinical and clinical development. Given that most of these products were not specifically designed to have selectivity toward biological targets, they should be considered as first-generation nanomedicines. , $2,7,8,9,10,40^{-1}$

\section{First generation of clinically approved nanomedicines}

Among a wide variety of nanosystems, only a few nanomedicines, such as Doxil ${ }^{\circledR}$ (Janssen Biotech Inc., Horsham, PA, USA), Myocet ${ }^{\circledR}$ (Sopherion Therapeutics Inc., Princeton, NJ, USA), DaunoXome ${ }^{\circledR}$ (Galen US Inc., Souderton, PA, USA), Depocyt ${ }^{\circledR}$ (Pacira Pharmaceuticals Inc., San Diego, CA, USA), Abraxane ${ }^{\circledR}$ (Celgene Corporation, Inc., Berkeley Heights, NJ, USA), Genexol-PM ${ }^{\circledR}$ (Samyang Biopharmaceuticals Corporation, Jongno-gu, Seoul, Korea), and Oncaspar ${ }^{\circledR}$ (Enzon Pharmaceuticals Inc., Bridgewater, NJ, USA), are approved for use in the treatment of cancer (Table 1).

Doxil, the first nanomedicine to secure regulatory approval by the FDA (for the treatment of Kaposi's sarcoma in 1995 and in Europe in 1997 with the brand name Caelyx ${ }^{\circledR}$ ), was obtained by encapsulating doxorubicin within liposomes. ${ }^{50,51}$ This nanoformulation consistently improved the pharmacokinetics and biodistribution profile of doxorubicin, thus facilitating a longer circulation half-life and maximizing drug accumulation in tumor tissue. However, despite clinical validation of this representative prototype of liposome technology in the treatment of metastatic breast cancer, ovarian cancer, and multiple myeloma, this class of nanovehicles generally fails to provide the controlled release and stability needed to control the kinetic profile and drug localization at the target tumor tissue. The non-PEGylated liposomal doxorubicin formulations, Myocet 
Table I Examples of nontargeted nanosystems in clinical use for anticancer therapy

\begin{tabular}{|c|c|c|c|c|}
\hline Name & Formulation & $\begin{array}{l}\text { Bioactive } \\
\text { compound }\end{array}$ & Indication & Status \\
\hline & Liposomes & & & \\
\hline DaunoXome ${ }^{\circledR}$ & Non-PEGylated liposomes & Daunorubicin & Kaposi's sarcoma & Approved \\
\hline Myocet $^{\circledR}$ & Non-PEGylated liposomes & Doxorubicin & Breast cancer & Approved \\
\hline Onco TCS ${ }^{\circledR}$ & Non-PEGylated liposomes & Vincristine & Non-Hodgkin's lymphoma & Approved \\
\hline \multirow[t]{2}{*}{ Depocyt $^{\circledR}$} & Non-PEGylated liposomes & Cytarabine & Leukemia & Phase III \\
\hline & & & Glioblastoma & Phase I/II \\
\hline Doxil ${ }^{\circledast} /$ Caelyx $^{\circledR}$ & PEGylated liposomes & Doxorubicin & $\begin{array}{l}\text { Breast cancer, ovarian } \\
\text { cancer, multiple myeloma, } \\
\text { Kaposi's sarcoma }\end{array}$ & Approved \\
\hline Thermodox ${ }^{\circledR}$ & PEGylated liposomes & Doxorubicin & Liver cancer, breast cancer & Phase III \\
\hline SPI-77 & PEGylated liposomes & Cisplatin & Ovarian cancer & Phase II \\
\hline \multirow[t]{2}{*}{ NL CPT } & PEGylated liposomes & Irinotecan & Glioma & Phase I \\
\hline & Polymeric nanoparticles & & & \\
\hline Genexol-PM $^{\circledR}$ & PEG-poly(lactic acid) & Paclitaxel & $\begin{array}{l}\text { Breast cancer, lung cancer, } \\
\text { ovarian cancer }\end{array}$ & Phase II \\
\hline \multirow[t]{2}{*}{ NKI05 } & PEG-poly(aspartic acid) & Paclitaxel & Gastric cancer & Phase I \\
\hline & & & Breast cancer & Phase III \\
\hline NK9II & PEG-poly(aspartic acid) & Doxorubicin & Various solid tumors & Phase II \\
\hline Opaxio $^{\mathrm{TM}}$ & PGA-paclitaxel & Paclitaxel & Lung cancer, ovarian cancer & Phase III \\
\hline CRLXIOI & PEG-cyclodextrin & Camptothecin & Non-small-cell lung cancer & Phase II \\
\hline NC-6004 & PEG-poly(glutamic acid) & Cisplatin & Pancreatic cancer & Phase II \\
\hline \multirow[t]{2}{*}{ ProLindac ${ }^{\mathrm{TM}}$} & HPMA & $\mathrm{DACH}-\mathrm{Pt}$ & Ovarian cancer & Phase II \\
\hline & Other & & & \\
\hline Abraxane $^{\circledR}$ & Albumin-based & Paclitaxel & Breast cancer & Approved \\
\hline Paclical $^{\circledR}$ & Micellar retinoid-derived & Paclitaxel & Ovarian cancer & Phase III \\
\hline NC-40I6 & Micellar PEG/polyamino acid & Oxaliplatin & Various solid tumors & Phase I/II \\
\hline Oncaspar ${ }^{\circledR}$ & PEG-L-asparaginase & $\begin{array}{l}\text { Asparagine } \\
\text { specific enzyme }\end{array}$ & $\begin{array}{l}\text { Acute lymphoblastic } \\
\text { leukemia }\end{array}$ & Approved \\
\hline
\end{tabular}

Note: DaunoXome ${ }^{\circledast}$ (Galen US Inc., Souderton, PA, USA); Myocet ${ }^{\circledast}$ (Sopherion Therapeutics Inc., Princeton, NJ, USA); Onco TCS ${ }^{\circledR}$ (Inex Pharmaceuticals Corp., Burnay, BC, Canada, and Enzon Pharmaceuticals Inc., Bridgewater, NJ, USA); Depocyt ${ }^{\circledR}$ (Pacira Pharmaceuticals Inc., San Diego, CA, USA); Doxil ${ }^{\circledR} /$ Caelyx ${ }^{\circledR}$ (Janssen Biotech Inc., Horsham, PA, USA / Janssen-Cilag Pty Ltd, Macquarie Park, NSW, Australia); Thermodox ${ }^{\circledR}$ (Celsion Corporation, Lawrenceville, NJ, USA); Genexol-PM ${ }^{\circledR}$ (Samyang Biopharmaceuticals Corporation, Jongno-gu, Seoul, Korea); Opaxio ${ }^{\mathrm{TM}}$ (Cell Therapeutics, Inc., Seattle, WA, USA); ProLindac ${ }^{\mathrm{TM}}$ (Access Pharmaceuticals Inc., Dallas, TX, USA); Abraxane ${ }^{\circledR}$ (Celgene Corporation, Inc., Berkeley Heights, NJ, USA); Paclical ${ }^{\circledR}$ (Oasmia Pharmaceutical AB, Uppsala, Sweden); Oncaspar ${ }^{\circledR}$ (Enzon Pharmaceuticals Inc., Bridgewater, NJ, USA).

Abbreviations: PEG, poly(ethylene glycol); HPMA, hydroxypropylmethacrylamide; DACH-Pt, diaminocyclohexane-platinum.

and DaunoXome, have been used for metastatic breast cancer and Kaposi sarcoma, respectively. ${ }^{52,53}$ Further, DepoCyt, a non-PEGylated liposomal nanocarrier loaded with cytarabine, was approved in 1999 for local intrathecal treatment of lymphomatous meningitis, and has now entered Phase III trials for leukemia and Phase I/II clinical trials for glioblastoma. ${ }^{52}$

Another therapeutic nanoformulation to secure clinical approval by the FDA (in 2005) is Abraxane, a co-condensate of albumin and paclitaxel..$^{53}$ In comparison with paclitaxel formulated using Cremophor ${ }^{\circledR}$ EL (the nononic surfactant used in the Taxol ${ }^{\circledR}$ formulation), Abraxane demonstrated significantly higher tumor response rates and longer times to tumor progression in patients with metastatic breast cancer. However, the utility of this technology is mainly limited to improving the therapeutic index of hydrophobic therapeutic agents (able to bind to albu$\mathrm{min}$ ) used in formulations with poorly tolerated vehicles.

More recently, in 2007 in Korea, another polymeric nanocarrier was approved for marketing, ie, Genexol-PM, a paclitaxel-loaded poly(lactic acid)-block-poly(ethylene glycol) micelle formulation, that was developed to avoid the need to use Cremophor EL. ${ }^{54}$ Genexol-PM led to an increase in the potency of paclitaxel in the treatment of breast and lung cancer, and is currently in Phase II clinical development in the US and Russia. With regard to PEGylated devices, Oncaspar, a combination of PEG-L-asparaginase, has recently been approved for the treatment of leukemia. ${ }^{52}$

\section{Liposomes and polymeric drug release nanoformulations in clinical development}

Despite the progress made in the clinical development of liposome-based nanocarriers, there are several issues that limit their wider consideration as a main drug delivery platform, including the difficulty in modulating their drug release in vivo, the limited amount of drug that can be loaded, possible oxidation of liposomal phospholipids, and the difficulty in 
maintaining a stable shelf-life. Otherwise, polymeric nanocarriers demonstrate similar to superior stability in vivo as compared to liposomes, have a high drug-loading capacity, and enable both controlled and triggered release of drugs. Therefore, polymeric-based nanomaterials have the potential to provide solutions for a range of problems in nanomedicine. ${ }^{40,55-58}$

The way forward for the clinical translation of controlledrelease polymeric nanoparticles was paved by the seminal work of Langer and Folkman in 1976, which demonstrated the controlled release of macromolecules from biodegradable polymers in a temporal manner. ${ }^{36}$ Moreover, a landmark paper by Langer et al in 1994 provided further evidence that diblock copolymers of biocompatible polymers with PEG can dramatically increase the controlled release and circulation half-life of polymeric nanoparticles. ${ }^{37}$ Because of their biocompatibility, biodegradability, and chemical and technologic flexibility, the polymeric materials most widely used for controlled drug release include PLA, poly(D,Llactide-co-glycolide) (PLGA), poly(caprolactone) (PCL), poly(glutamic acid), N-(2-hydroxypropyl)-methacrylate copolymers, and poly(amino acids). ${ }^{56}$

\section{“Drug-targeting" strategies}

Since Paul Ehrlich, considered the "father of chemotherapy", suggested the concept of a "magic bullet", ie, "a drug that selectively attaches to diseased cells but is not toxic to healthy cells" approximately a century ago, ${ }^{59}$ a great deal of interest has been channeled in this direction, focused particularly on the treatment of cancer. ${ }^{60}$ Several strategies can be exploited to reach a putative biological target, and include passive drug targeting, active drug targeting, active drug targeting to endothelial cells, and triggered drug delivery (Figure 3Aa-Dd). ${ }^{6-8,10,12,40,56-58}$

\section{Passive drug targeting}

The majority of nanosystems show prolonged blood circulation times in vivo and accumulate at particular sites simply due to the balance between vascular hemodynamic forces
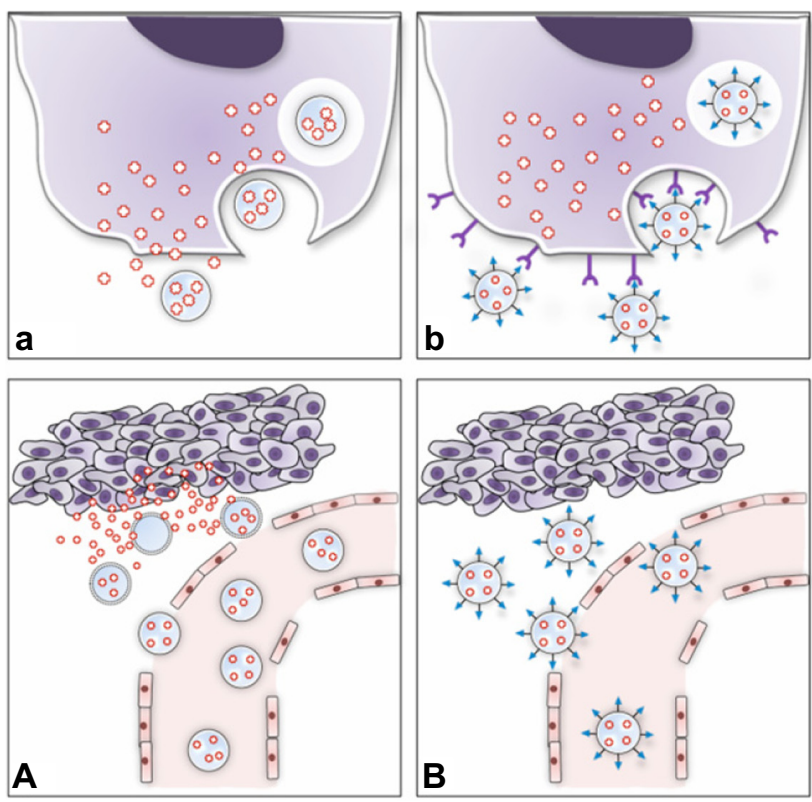

Passive drug targeting

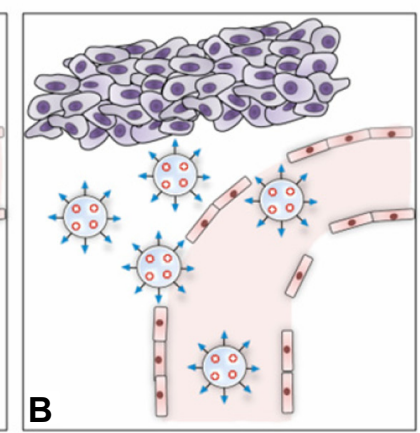

Active drug targeting
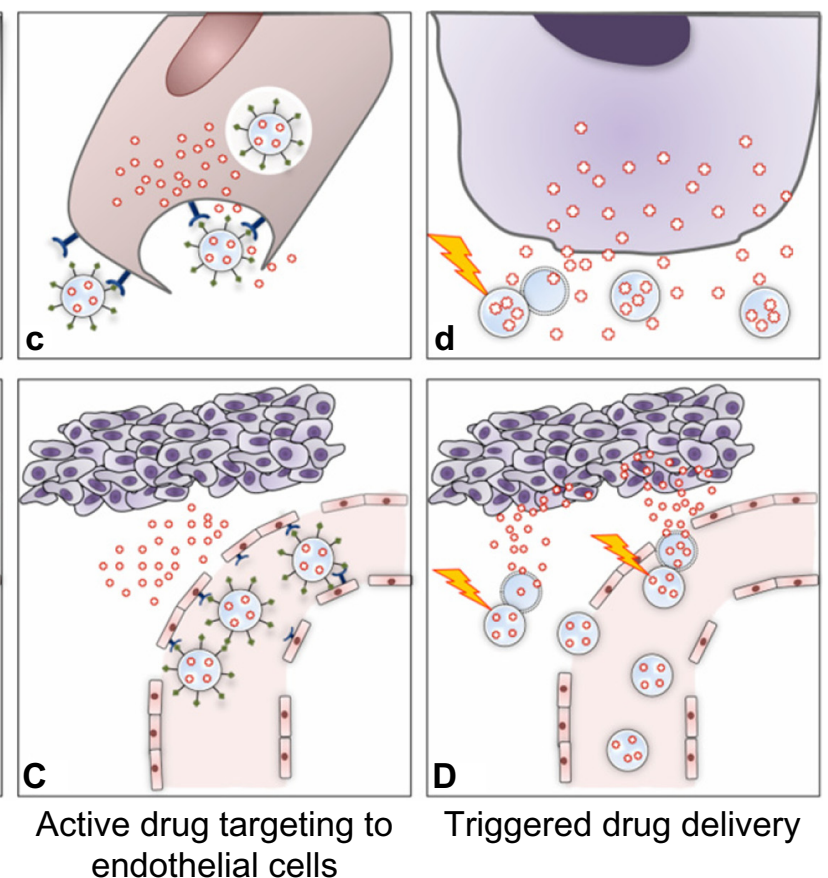

Figure 3 Strategies adopted for drug targeting and localization of nanosystems to tumor cells and tissues.

Notes: (A) Passive drug targeting. Circulating nanoparticles passively extravasate in solid tumor tissue via the enhanced permeability of blood vessels, ie, through the disorganized and leaky vasculature surrounding the solid tumor coupled with the absence of lymphatic drainage, and preferentially accumulate in tumor cells (the EPR effect). (a) The drug is released into the extracellular matrix and diffuses through the cells and tissue. (B) Active drug delivery. Once nanoparticles passively extravasate and concentrate in the target tissue via the EPR effect, the presence of ligands grafted onto the nanoparticle surface enable active targeting of the nanoparticles to receptors that are overexpressed on tumor cells or tissue, resulting in enhanced uptake and internalization via receptor-mediated endocytosis. (b) Tumor-specific ligands on the nanoparticles bind to cell surface receptors, triggering internalization of the nanoparticles into the cell through endosomes on which, due to an internal acidic $\mathrm{pH}$, the drug is released from the nanoparticles and diffuses into the cytoplasm. (C) Active drug targeting to endothelial cells. Nanoparticles can be targeted to bind to angiogenic endothelial cell surface receptors with the aims of: enhancing drug accumulation in the tumor endothelium, thereby inhibiting growth of blood vessels supplying the tumor rather than inhibiting tumor cells per se (c); and improving delivery of chemotherapeutic agents to tumor cells via the EPR effect with the potential to act synergistically in targeting both the vascular tissue and tumor cells. (D) Triggered drug delivery by stimuli-sensitive nanomedicines. Nanoparticles passively accumulate in the tumor via the EPR effect. After localization at the target site or while circulating in the tumor vasculature (d), the nanoparticles can be activated by external stimuli (eg, hyperthermia, light, magnetic fields, ultrasound) that induce release of the payload drugs. Images adapted from J Control Release, I6I(2), Lammers T, Kiessling F, Hennink WE, Storm G, Drug targeting to tumors: principles, pitfalls and (pre-) clinical progress, 175-187, Copyright 2012, with permission from Elsevier. ${ }^{10}$

Abbreviation: EPR, enhanced permeability and retention. 
and diffusion mechanisms. Passive drug targeting is widely exploited in chemotherapy because nanoparticles circulating in the bloodstream can localized to neoplastic tissues through the well known enhanced permeation and retention effect. ${ }^{61,62}$ Anatomically, tumor microvasculature is leaky and characterized by abnormal branching and enlarged interendothelial gaps, with associated breakdown of tight junctions between endothelial cells and a disrupted basement membrane. ${ }^{63}$ These large gaps between endothelial cells facilitate the extravasation of particulate material from the surrounding vessels into the tumor (Figure 3A). ${ }^{64}$ Therefore, in contrast with the vasculature in healthy tissues, the poorly differentiated vasculature of immature tumors, with pores smaller than about $400 \mathrm{~nm}$, allows for extravasation and selective accumulation of nanoparticles in the tumor interstitium via a passive targeting mechanism (Figure 3A). ${ }^{42,65}$ Importantly, it should be borne in mind that the enhanced permeation and retention effect is a very heterogeneous phenomenon, varying dramatically from tumor to tumor and from patient to patient. ${ }^{66}$ For example, even considering a single tumor model, there are many differences with regard to vascular permeability, where particles with a diameter $>200-300 \mathrm{~nm}$ are able to extravasate, whereas in another part of the same tumor, molecules only a few nanometers in size may have difficulty entering the interstitium.

Most passive targeting nanosystems have a surface coated with PEG for biocompatibility and "stealth" purposes. ${ }^{42-44}$ Importantly, it should be noted that increased hydrophilicity on the nanoparticle surface can impede its uptake by cancer cells, thereby hampering efficient drug delivery to tumors by passive targeting nanoparticles. ${ }^{42,47,67}$ However, PEG-based block copolymers have resulted in the clinical translation of numerous passive targeting polymeric nanoparticles, including the abovementioned Genexol-PM, as well as SP1049C, NK911, and other prototypes now entering early clinical trials for the treatment of a variety of cancers (Table 1).

SP1049C is a Pluronic polymeric micelle nanoparticle carrying doxorubicin, and is currently undergoing Phase II trials in metastatic cancer of the esophagus refractory to standard chemotherapeutic protocols. ${ }^{68}$ Another example of a polymeric nanocarrier that acts via a passive targeting mechanism is NK911, a micellar nanoparticle comprising PEG, doxorubicin, and poly(aspartic acid), currently in Phase II clinical development for various types of cancer. ${ }^{69}$ Moreover, Opaxio ${ }^{\mathrm{TM}}$ (formerly Xyotax $^{\mathrm{TM}}$ ), also a passively targeted paclitaxel/poly(L-glutamic acid) nanoconstruct, is proving effective in ovarian tumors, ${ }^{70,71}$ and CRLX101 (formerly IT-101), a camptothecin-cyclodextrin polymer conjugate, is showing enhanced pharmacokinetic efficacy in both preclinical and clinical studies. ${ }^{72}$ Further conjugated polymer-drug nanotherapeutics, such as NC-6004 [a cisplatin-incorporated PEG-poly(glutamic acid) block copolymer micellar formulation] and ProLindac ${ }^{\mathrm{TM}}$ (a diaminocyclohexane-platinum hydroxypropylmethacrylamide prodrug), are in late-stage clinical trials. ${ }^{10}$ In addition, Paclical ${ }^{\circledR}$, a micellar formulation of paclitaxel, has recently received orphan drug designation by the FDA, and is currently in Phase III trials for ovarian carcinoma. ${ }^{73}$ Passive targeting lipid nanocarriers are also moving into the advanced stages of clinical trials, and intensive effort is being made to get these systems into clinical practice (Table 1). Promising liposomal nanomedicines in clinical trials include Thermodox ${ }^{\circledR}$, a thermosensitive liposomal doxorubicin formulation that releases the active drug at temperatures around $39^{\circ} \mathrm{C}$, and is currently being tested in Phase III trials together with radiofrequency ablation in patients with hepatocellular carcinoma. ${ }^{74}$ Finally, SPI-77, a PEGylated liposomal formulation of cisplatin, is in Phase II trials for patients with recurrent epithelial ovarian cancer, ${ }^{75}$ and a nanoliposomal formulation of irinotecan (CPT-11) is in Phase I trials for glioma. ${ }^{76}$ Several liposomal formulations carrying two different types of chemotherapeutics, such as cytarabine and daunorubicin, are also reported to be in the process of clinical development. ${ }^{77}$

\section{Active targeting as a strategy for developing site-directed nanoparticles}

Passive targeting strategies have shown several limitations, so a considerable amount of work is now underway investigating active targeting nanoformulations that can maximize their accumulation at sites of interest. ${ }^{7,8,10}$ In this respect, polymeric nanoparticles allow for versatile modification possibilities, and can act as functional platforms for the assembly of well defined multifunctional structures. ${ }^{40,56-58,78}$ In fact, slight variations in polymeric composition as well as ligand surface functionalization can facilitate the targeting ability of nanoparticles in biological systems. Active drug targeting involves the use of a variety of affinity ligands to direct the binding of nanoparticles to many biological targets, largely represented by antigens, that are differentially overexpressed both in the plasma membrane and in diseased tissue (Figure 3B and b). ${ }^{40,79}$ This approach can be used for controlled drug release applications, where the drug is released into either the extracellular or intracellular compartment. In the latter process, internalization of nanoparticles by receptor-mediated endocytosis can occur through several pathways that lead to endosome formation, and ultimately 
allow for generation of lysosomes (Figure 3b). ${ }^{56,80}$ Since the primary role of the targeting ligand is to enhance the uptake of nanoparticles into target cells, cell internalization by active targeting nanoparticles is postulated to improve the therapeutic efficacy as compared with nontargeted nanoparticles. ${ }^{56-58}$ This suggests that while the biodistribution would be strictly related to the colloidal properties of the nanoparticles, the targeting ligand is important for enhancing both cell recognition and cell uptake at target sites. While the potential benefit of active targeting nanoparticles seems to be widely accepted, this technology has resulted in only a few clinically validated nanoproducts (Table 2). ${ }^{10,40,58}$

\section{Active drug targeting to endothelial cells and triggered drug delivery}

Several important pitfalls in active targeting of drugs to tumor cells have been identified, including: poor tumor penetration by nanomedicines; the complexity of predicting the potential usefulness of an active drug targeting approach for a specific application; difficulty in managing nanoformulations that are too complex in design; the not always favorable implementation of nanomedicine formulations in clinically consistent combination regimens; and the low level of knowledge concerning the biological and (patho)physiologic principles of tumor progression and related treatment. ${ }^{10}$ Although this approach has potential in controlled and targeted drug delivery, the failure of translation of a great number of targeted nanomedicines at the clinical level can likely be attributed to the inability of targeted nanoparticles to overcome a succession of membrane layers as biological barriers, represented by pericyte-based, smooth muscle cell-based, and fibroblastbased cell layers between endothelial and cancer cells, as well as a plethora of cellular processes and anatomic tumor issues (ie, the high cellular density within solid tumors and high interstitial fluid pressure) that represent important obstacles for reaching the target tumor interstitium. ${ }^{4,6,8-10,56}$

To address these issues, a variety of vascular-targeted nanoformulations have been designed and evaluated (Figure 3C and c). ${ }^{8-10,56}$ Example of ligands targeting angiogenic endothelium are the linear and cyclic derivatives of an oligopeptide containing the Arg-Gly-Asp (RGD) sequence, that binds to target endothelium via integrin receptors. ${ }^{81,82}$

Another targeting strategy capable of generating innovative nanoformulations focuses on the possibility that a nanosystem can be triggered to release its contents (ie, the payload drug) on exposure to external and confined stimuli, such as light, heat, ultrasound, and magnetic fields, in order to maximize drug release at the pathologic site (Figure 3D and $3 \mathrm{~d}) .{ }^{10}$ The above mentioned product, Thermodox, a temperature-sensitive doxorubicin-PEGylated liposome, is an interesting example of stimuli-responsive nanomedicine. ${ }^{74}$ Despite the clinical potential of these nanoformulations, there are still several important limitations to their extensive clinical development, that include manufacturing difficulties and problems with managing the way in which they respond to stimuli-responsive drug release. However, much effort has been made to improve the stimuli-responsiveness of these tumor-targeted nanotherapeutics, and the development of

Table 2 Tumor-targeted nanomedicines in clinical development

\begin{tabular}{|c|c|c|c|c|c|}
\hline Name & Formulation & Targeting ligand & Bioactive compound & Indication & Status \\
\hline & Liposomes & & & & \\
\hline CALAA-0I & $\begin{array}{l}\text { Cyclodextrin-based NP } \\
\text { containing anti-RRM2 }\end{array}$ & Transferrin & siRNA & Various solid tumors & Phase I \\
\hline MBP-426 & Liposomes & Transferrin & Oxaliplatin & $\begin{array}{l}\text { Gastroesophageal } \\
\text { adenocarcinoma }\end{array}$ & Phase II \\
\hline MCC-465 & PEGylated liposomes & $\begin{array}{l}F\left(a b^{\prime}\right) 2 \text { fragment of human } \\
A b \text { GAH }\end{array}$ & Doxorubicin & $\begin{array}{l}\text { Metastatic stomach } \\
\text { cancer }\end{array}$ & Phase I \\
\hline SGT53 & Liposomes & $\begin{array}{l}\text { Anti-transferrin receptor } \\
\text { single-chain Ab fragment } \\
\text { (TfRscFv) }\end{array}$ & p53 gene & Solid tumors & Phase I \\
\hline \multirow[t]{2}{*}{ C225-ILS-DOX } & PEGylated liposomes & $\begin{array}{l}\text { Antigen-binding fragments } \\
\text { (Fab) of cetuximab }\end{array}$ & Doxorubicin & Advanced solid tumors & Phase I \\
\hline & Polymeric nanoparti & & & & \\
\hline $\begin{array}{l}\text { BIND-0I4 } \\
\text { (Accurins }{ }^{\mathrm{TM}} \text { ) }\end{array}$ & PEGylated PL(G)A & Small molecule & Docetaxel & Solid tumors & Phase II \\
\hline Atu027 & Liposomes & Protein kinase N3 & siRNA & Solid tumors & Phase I \\
\hline C-VISA-BikDD & Liposomes & Proapoptotic gene & BikDD plasmid DNA & Pancreatic cancer & Phase I \\
\hline
\end{tabular}

Note: Accurins ${ }^{T M}$ (BIND Therapeutics, Inc., Cambridge, MA, USA).

Abbreviations: NP, nanoparticles; PEG, poly(ethylene glycol). 
more effective nanoparticles for triggered drug delivery is expected in the near future. ${ }^{83,84}$

\section{Methods and ligands used for targeted drug delivery}

In the course of the last decade, several strategies involving conjugation of targeting ligands to the surface of nanoparticles have been developed. ${ }^{38,40,45,55-57}$ As far as the targeting approach is concerned, one key issue relies on the choice of optimal targeting ligands, possibly by balancing their stoichiometry in comparison with the antibiofouling surface of nanoparticles. More specifically, two important ligand properties, ie, affinity and density, can have a key role in effective targeting of nanoparticles to the cell surface membrane. Again, the ligand binding affinity is the result of the equilibrium between enthalpic advantages (for ligandreceptor interaction) and entropic losses (stretching, flexibility, or compressibility of the nanosystem). For example, greater ligand density does not necessarily lead to a higher intracellular concentration, given the decrease in "stealth" surface characteristics. Moreover, although the uptake of nanoparticles usually increases with an increasing $+/-$ charge ratio of nanoparticles (in terms of zeta potential values), an excess positive charge can induce toxicity and promote an immunologic reaction. Therefore, the optimal ligand density and charge on the nanoparticle surface should be investigated on a case-by-case basis. However, a wide range of chemical modifications is now available to attach targeting ligands to nanoparticles. ${ }^{55-57,85}$

Conventional methods of preparing targeted nanoparticles involve two typical options, ie, the "postcoupling" and "precoupling" approaches. ${ }^{55-57}$ The first approach involves bioconjugation of the preformed nanoparticle core with targeting ligands to the nanoparticle surface. This postcoupling approach for attaching affinity ligands does not allow for fine-tuning of the ligand-nanoparticle surface density for optimal efficacy. Conversely, most effective chemical strategies include preconjugation of a targeting ligand after nanoparticle formation followed by nanoformulation (ie, the precoupling method). For example, effective self-assembly of prefunctionalized triblock polymers (ie, copolymer-PEG ligands), accomplished in the pioneering work of Farokhzad and Langer ${ }^{86,87}$ demonstrated the possibility of developing more homogenously targeted nanoparticles capable of controlling ligand-to-nanoparticle ratios and preventing a heterogenous distribution of ligands on the surface of nanoparticles. Summarizing this information, it is clear that a combination of several "unpredictable" factors, including the nature of the targeting ligand, the size, shape, and composition of the targeted nanosystem, as well as the balance of the ligand-nanoparticle surface ratio, can synergistically contribute to the efficiency of binding between targeted nanoparticles and their target cells, and directly affect the biological profile in vivo. Therefore, all these aspects should be investigated in a rational fashion when developing a nanosystem.

Using different approaches, nanoparticles can be functionalized using a variety of targeting ligands that include monoclonal antibodies or their fragments, proteins, or peptides, nucleic acid ligands (such as aptamers), and small molecules, the characteristics of which are outlined in the following sections and summarized in Figure $4 .{ }^{88}$

\section{Monoclonal antibodies}

Monoclonal antibodies have for several years been the preferred class of targeting ligands, and engineered monoclonal antibodies, preferably one capable of evading the immune system, have been widely used in the development of targeted nanoparticles. Current development of monoclonal antibodies has led to chimeric and humanized derivatives to enable modulation of their immunogenicity. The ability of engineered monoclonal antibodies to target and interfere with cellular processes has been demonstrated by the success of several monoclonal antibody therapeutics. ${ }^{89,90}$ Some of these, including rituximab and trastuzumab, have been conjugated to PLA nanoparticles, resulting in nanoconjugates that show a significant increase in the rate of particle uptake compared with their nontargeted counterparts. ${ }^{91}$ However, despite the intense effort undertaken for their development, monoclonal

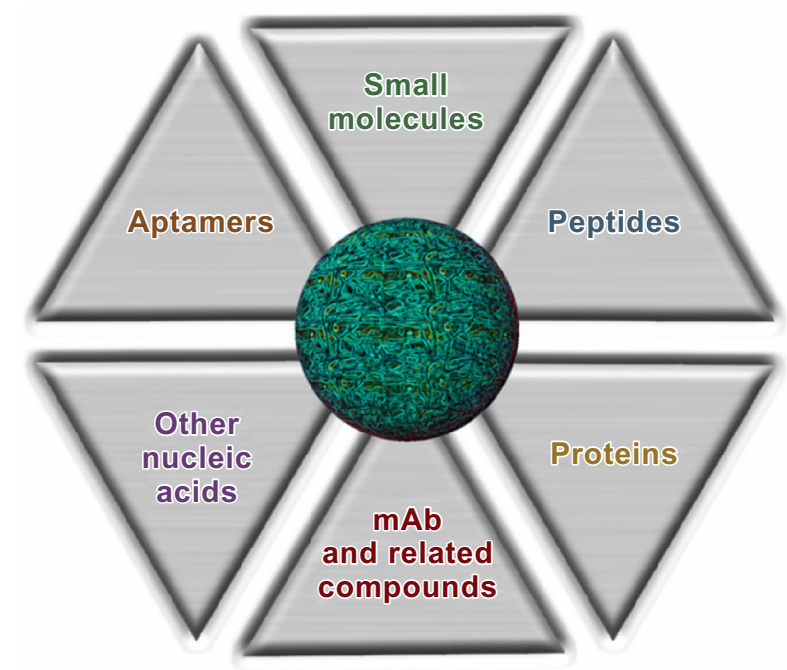

Figure 4 Types of ligands used for targeted nanoparticles. Abbreviation: mAb, monoclonal antibody. 
antibodies still have a number of limitations, including their large size, requirement for extensive optimization through molecular engineering technologies, difficulty in managing nanoparticle scale-up and manufacturing, and potentially immunogenic characteristics, resulting in rapid nanoparticle clearance. Due to these problems in using monoclonal antibodies, there is increasing interest in engineering and using antibody fragments as targeting molecules to retain the high antigen binding specificity of the parent antibodies, but with less immunogenicity and a smaller size, and thus obtain monoclonal antibodies that are better suited for molecular targeting. These include the Fab fragments, single chain variable fragments (scFv), minibodies, diabodies and nanobodies. ${ }^{2,92}$

\section{Aptamers}

Aptamers are small, single-stranded RNA or DNA sequences of oligonucleotides that can be designed as ligands capable of binding to targets with high sensitivity and specificity. ${ }^{93,94}$ They are small in size (usually approximately $15 \mathrm{kDa}$ ), and have less immunogenicity with respect to monoclonal antibodies or other macromolecules, leading to better stability and biodistribution. ${ }^{95,96}$ Such biomolecules fold by intramolecular interactions into unique three-dimensional conformations and topologies, having the ligand-binding characteristics needed for efficient target (receptor/antigen) affinity. Selective aptamers, with binding affinity toward a specific target, are currently being identified by an in vitro chemical process known as "systemic evolution of ligands by exponential enrichment" (SELEX). ${ }^{97}$ In this process, which can be easily scaled up at a relatively low cost, a library of ten random oligonucleotides is enriched in order to identify the prototype with the highest affinity and specificity. Using this technology, Langer and Farokhzad designed and developed customized controlledrelease nanoparticles loaded with docetaxel and having tumortargeting capability toward prostate-specific membrane antigen (PSMA). ${ }^{40,86,87,98,99}$ Targeted nanoparticles have been obtained by decorating their shell surface with RNA A10-aptamer to bind PSMA, ${ }^{100-102}$ a clinically validated tumor marker that is not only overexpressed on the surface of prostate cancers but also on the neovasculature of many solid tumors. This approach constituted the proof-of principle for development of the first targeted polymeric nanoformulation (ie, BIND-014), which is now approved for clinical use (see below).

\section{Proteins and peptide-based targeting molecules}

Several endogenous proteins capable of selectively binding to specific receptors on membrane cells can be used for targeting purposes via receptor-mediated endocytosis. ${ }^{103}$ For example, transferrin, an iron-transporting protein that binds specifically to transferrin receptors (TfR) located on the cell surface, has been used to transport nanoparticles into different types of cells. ${ }^{103,104}$ However, their effectiveness for targeting purposes may be limited by their immunogenicity and susceptibility to early clearance. The target receptors for these proteins are also commonly expressed on various types of nontargeted cells, which can lead to unwanted off-target effects.

Peptide-based ligands are emerging as attractive alternative targeting molecules due to their small size, high stability, and relatively low immunogenicity as compared with many proteins. Identification of specific targeting ligands has usually derived from previously or newly focused peptide phage/bacterial/plasmid peptide display libraries, as well as use of newer and easier screening technologies. ${ }^{105}$ Like monoclonal antibodies and aptamers, peptides can bind to several molecular targets with a high degree of affinity and specificity, and are easy to manufacture by conjugation to polymers, lipids, and various nanoparticle surfaces. ${ }^{106,107}$

\section{Small molecules}

Small molecules, usually defined as low molecular weight organic molecules with a molecular weight $<500 \mathrm{Da}$, constitute a promising class of targeting molecules because of their small size, high stability, chemical management, and low production cost. ${ }^{57,58,108}$ Some further advantages of small molecules as targeting ligands include: availability of suitable facile coupling chemical methods for their conjugation; the possibility to modulate ligand densities and charge on nanoparticle surfaces, since these parameters can affect stability, size, and morphology, as well as targeting efficiency; availability of a wide range of targeting ligands with variable physicochemical properties and a variety of functional groups; fewer immunogenic effects in vivo; and reproducible, scalable, and economical manufacturing. Among the large number of small molecules identified as potential targeting ligands, folic acid has been one of the most extensively studied and used ligands in targeted drug delivery. ${ }^{108,109}$ Due to its high affinity for folate receptors, which are overexpressed in many types of tumor cells, the vitamin folate has also been used to deliver drug conjugates and many drug delivery systems (ie, liposomes, polymeric nanoparticles, dendrimers) to selectively accumulate drugs into cancer cells using folate receptor-mediated endocytosis. ${ }^{110}$

Pomper et al have previously identified small hydrophilic molecules from a series of urea-based PSMA inhibitors as novel diagnostic agents capable of targeting the PSMA 
receptor in prostate cancer cells with affinity and specificity similar to that of antibodies and aptamers. ${ }^{11-113}$ Interestingly, a small molecule belonging to this class of molecules, ie, the pseudomimetic dipeptide $2-\{[(5$-amino-1-carboxypentyl) carbamoyl]amino pentanedioic acid, ${ }^{111}$ already used by Sechi et al for development of (-)-epigallocatechin 3-gallateloaded PSMA-targeted nanoparticles ${ }^{114}$ and by Chandran et al for preparation of docetaxel-PLA/PCL-based targeted nanoparticles, ${ }^{115}$ was also used as a targeting ligand in the development of BIND-014. ${ }^{116}$

Concerning other small molecules that could be used as targeting ligands, carbohydrates, ie, sugar-based compounds, have also gained attention due to their low molecular weight, high availability and biocompatibility, and ease of production. Several of these carbohydrate ligands target membrane carbohydrate-binding proteins (membrane lectins) that are differentially expressed on the cellular and intracellular membranes, and some carbohydrates, such as mannose, glucose, galactose, and their derivatives, have been considered as potential ligands for carriers in cell-selective drug delivery. ${ }^{117,118}$

The clinical translation of the above-described technologies has ushered in a new era in the development of innovative therapeutic nanoparticles that are capable of targeting and controlled release, also considering the possibility of codelivery of multiple active agents.

\section{Clinical development of targeted nanoparticles}

To date, only three targeted liposomes and four targeted polymeric nanoparticles have progressed in clinical development (Table 2). The prototype that reach clinical trials was MCC-465, a new generation of liposome-encapsulated doxorubicin with a surface covered by both PEG and antigenbinding fragments $\left[\mathrm{F}\left(\mathrm{ab} \mathrm{b}^{\prime}\right) 2\right]$ to confer immune shielding and targeting, respectively, that is used in the treatment of human stomach cancer. ${ }^{119}$ Another site-directed liposome is SGT53-01, a TfR-functionalized nanoformulation containing a chain antibody fragment (TfRscFv) as the targeting ligand. It has been designed to carry the p53 tumor suppressor gene to cancer cells, and is currently undergoing Phase I clinical trials in combination with doxorubicin for the treatment of solid tumors. ${ }^{120}$ The third site-specific liposomal nanocarrier is MBP-426, a TfR-targeted liposome encapsulating oxaliplatin, that has been used clinically for the treatment of a variety of solid tumors. ${ }^{121,122}$

With regard to targeted polymeric nanoformulations, CALAA-01 was the first nanotherapeutic to reach clinical development for siRNA delivery in $2008 .{ }^{123}$ This nanosystem consists of TfR-targeted cyclodextrin-based PEGylated nanoparticles containing siRNA, and is capable of reducing expression of the M2 subunit of ribonucleotide reductase. The safety of CALAA-01 was evaluated in a Phase I clinical trial by intravenous administration to adults with solid tumors refractory to standard of care therapies. ${ }^{124}$

More recently, a new antiepidermal growth factor receptor nanoimmunoliposome loaded with doxorubicin (ie, C225ILS-DOX) entered Phase I investigation for the treatment of solid tumors. ${ }^{125}$ Fab fragments of the chimeric monoclonal antibody cetuximab (C225, Erbitux) were covalently conjugated to the liposome membrane to target nanoparticles against tumor cells overexpressing EGFR (Table 2). ${ }^{126}$

Focusing on the first clinically tested targeted nanomedicine, a major effort has made on BIND-014 (Table 2), ${ }^{116}$ a new docetaxel formulation developed by a team led by Langer and Farokhzad at the Massachusetts Institute of Technology, Harvard Medical School, and BIND Therapeutics, as a programmable nanomedicine that recently entered Phase II clinical testing for the treatment of patients with solid tumors (Figure 5). The development of BIND-014, along with its pharmacobiologic profile and potential clinical outcomes, is discussed in the following sections.

\section{Pharmacology, mode of action, and pharmacokinetics of BIND-0 I 4 Development of BIND-0I4}

The clinical translation of a targeted drug delivery nanosystem is strictly related to an optimal and synergistic combination of several physicochemical features, including polymer type, size, surface charge, hydrophilic/hydrophobic balance, ligand type and density, drug properties (type, solubility, loading, kinetics), and in vivo release capability (ie, tissue penetration, navigation across membrane layers and biological barriers, immune evasion or "stealth" characteristics, and cellular binding, uptake, and internalization processes)..$^{8,40,56,58,116}$

Considering these issues, BIND Biosciences (now BIND Therapeutics) developed customized controlled-release nanoparticles by using new high-throughput technology to achieve multifactorial optimization of polymeric nanosystems. The history of this project started with the pioneering work of Langer and Farokhzad that led to proof-of-concept for the development of innovative drug delivery vehicles composed of several safe or FDA-approved materials as biocompatible polymers (ie, PLA, PLGA, and PEG) and aptamers to target PSMA. ${ }^{40,85,86}$ Such nanoparticles were loaded with docetaxel, a semisynthetic taxane and one of the most commonly 


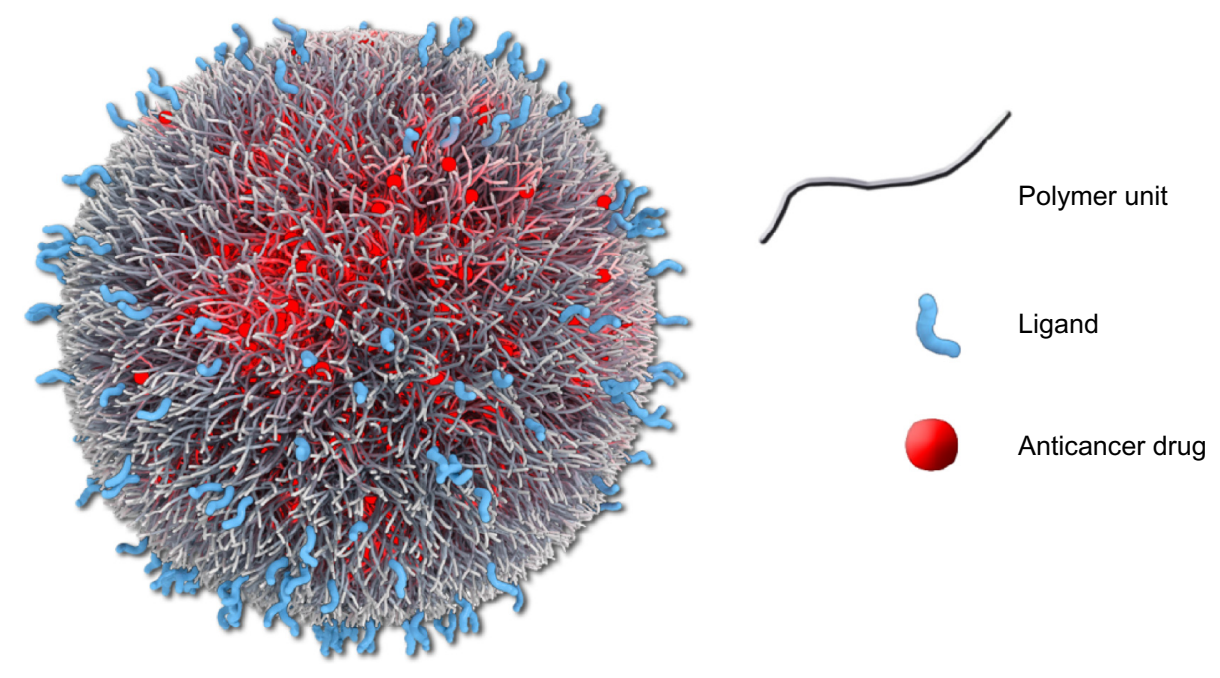

Figure 5 Graphic representation of BIND-0I4 composed of a biodegradable and hydrophobic PLA polymeric core and a hydrophilic PEG corona decorated with smallmolecule (a pseudomimetic PSMA-directed dipeptide) targeting ligands, and a semisynthetic taxane (docetaxel) as an encapsulated anticancer drug. Note: Images adapted with the permission of BIND Therapeutics (by Gael McGill), Harvard Medical School, and Digizyme Inc. Abbreviations: PLA, poly(lactic acid); PEG, poly(ethylene glycol); PSMA, prostate-specific membrane antigen.

used cancer chemotherapy drugs. ${ }^{127-129}$ More specifically, a hydrophilic spacer (ie, PEG) was incorporated into the outer shell of the nanoparticle in order to achieve minimal self-self and self-nonself interaction, to escape capture by the reticuloendothelial system, and to reduce opsonization, thus enabling "stealth" properties for immune evasion. ${ }^{42,43,130}$ The tumor-targeting capability of these nanoparticles was obtained by "decorating" the shell surface with RNA A10aptamer (then replaced with a small molecule urea-based PSMA inhibitor) to bind PSMA. ${ }^{40}$ The biological results demonstrated that the encapsulated drug efficiently interacted with the tumor and selectively delivered docetaxel to prostate cancer cells. ${ }^{40,86,87}$

Optimization of such construct to BIND-014 was done by adopting a modular self-assembly approach involving use of prefunctionalized polymeric materials to generate a combinatorial library of more than 100 self-assembled polymeric targeted nanoparticles of varying composition. This library was designed by introducing physicochemical diversity into each nanoparticle via a focused number of components, and by systematically varying both the nanoformulation and the critical process parameters (Figure 6). ${ }^{116}$

These targeted docetaxel-loaded nanoparticles were constructed with the above mentioned ternary structure and comprise a biodegradable copolymeric core (PLA or PLGA and PEG), a pseudomimetic dipeptide as a PSMAtargeting ligand, and docetaxel as the payload anticancer drug. Importantly, because scale-up of production for nanoformulations is a huge challenge that has precluded many prototypes going from bench scale experiments to clinical trials, several key factors that can contribute to the translation of this nanotherapeutic platform were identified. These include the use of FDA-approved polymeric materials that have already been validated for preparation of pharmaceutical products and biomedical application. Moreover, a major goal has been reached by the development of a robust, scalable, and continuous process for efficient nanoparticle formation by using a suitable model (encapsulation of a hydrophobic cancer-preventive compound) to obtain high drug loading and encapsulation efficiency. For example, instantaneous mixing of materials during nanoformulation provides the opportunity to achieve the higher drug loading parameter. ${ }^{116}$ Therefore, understanding of the fundamental thermodynamics and kinetics to optimize production of the nanoparticles in a repeatable and scalable manner constitutes a key aspect to enable manufacturing of clinical candidate nanoparticles in the kilogram quantities needed for clinical use. The evolution of this process resulted in BIND-014, the first targeted and controlled release polymeric nanoparticle for cancer chemotherapy to reach clinical Phase I trials in January 2011. ${ }^{116,131}$

\section{Pharmacobiologic profile of BIND-0I4}

Overall, BIND-014 has been demonstrated to be up to 10 -fold more effective in delivering docetaxel to tumors with respect to an equivalent dose of free drug in multiple animal models, with no increase in toxicity. ${ }^{116}$ In particular, as far as tissue biodistribution is concerned, intravenous administration of nanoparticles in in vivo experiments in rats showed a blood circulation half-life of about 20 hours, 


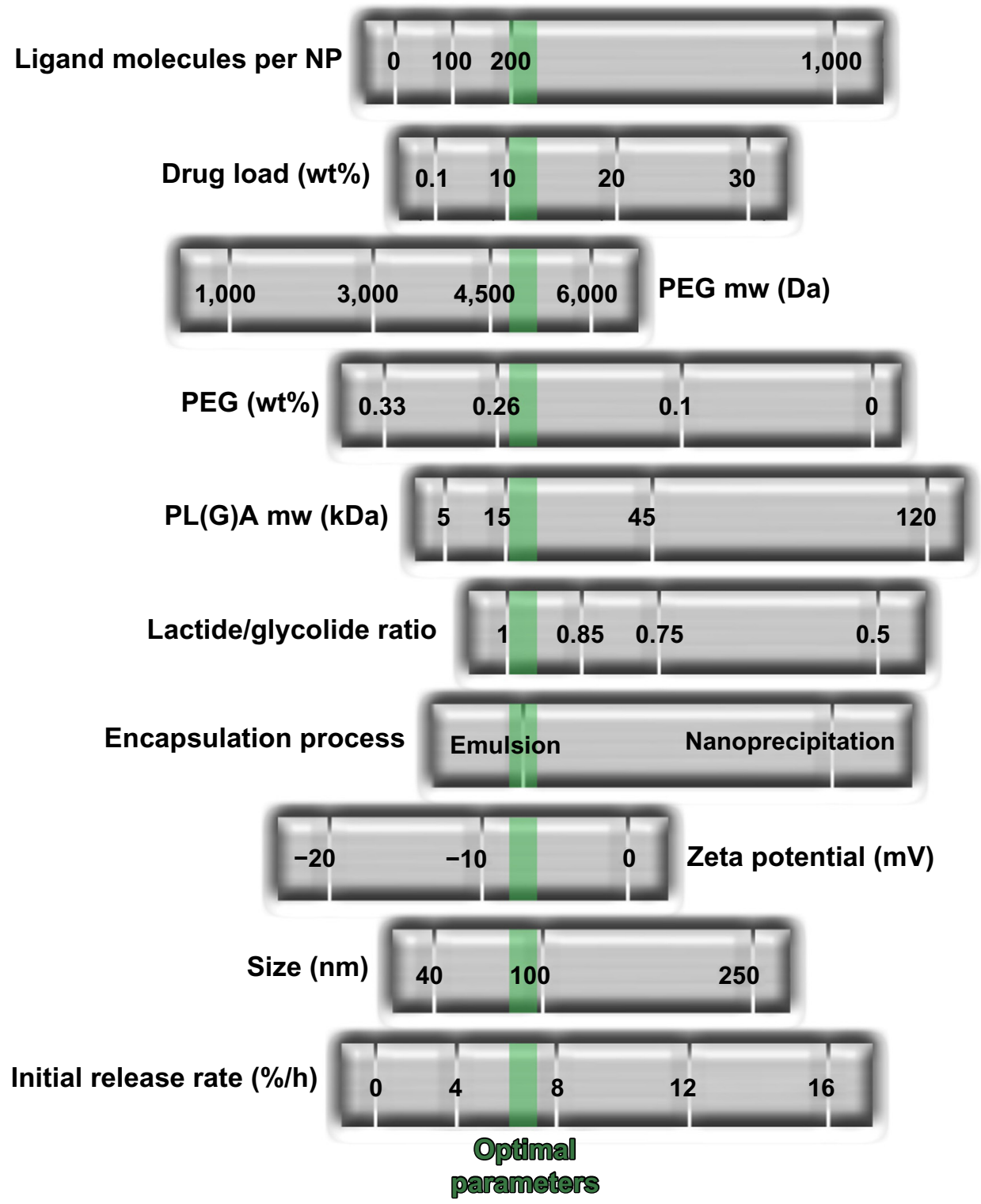

Figure 6 Development of BIND-0I 4 by high-throughput technology. Multifactorial optimization of polymeric nanosystems was performed by varying a range of formulation process parameters and physicochemical nanoparticle properties. The green dotted line indicates optimized nanoparticle parameters.

Notes: Molecular weight expressed in Dalton ( $\mathrm{Da})$; zeta potential expressed in millivolts $(\mathrm{mV})$; diameter expressed in nanometers (nm); initial release rate calculated by mass flow per unit time $(\% / \mathrm{h})$. From Hrkach J, Von Hoff D, Mukkaram Ali M, et al. Preclinical development and clinical translation of a PSMA-targeted docetaxel nanoparticle with a differentiated pharmacological profile. Sci Transl Med. 2012;4(I28):I28ra I39. ${ }^{116}$ Adapted with permission from AAAS.

Abbreviations: NP, nanoparticles; PEG, poly(ethylene glycol); PL(G)A, poly(lactic acid) or poly(D,L-lactide-co-glycolide).

with higher plasma docetaxel concentrations relative to those of the free drug (as formulated in Taxotere ${ }^{\mathrm{TM}}$ ), where encapsulation prevents rapid distribution of docetaxel from the plasma compartment to tissues (lower accumulation of nanoparticles being detected in liver and bone marrow with respect to plasma). The in vitro release and in vivo pharmacokinetic results also demonstrated that the large amount of docetaxel released from these polymeric nanoprototypes resulted in a long blood circulation time, with sustained release of the drug, leading to an increase of up to 1,000 -fold in the docetaxel rate in the plasma, available to reach tumor sites with respect to the conventional docetaxel formulation (ie, Taxotere). ${ }^{116}$

Moreover, toxicologic characterization of the composition of free nanoparticles (without drug), targeted nanoparticles, and the respective nontargeted nanoparticles, has been done in a single-dose tolerability study in rats. The results indicated that the ingredients used in the nanoformulation are in general well tolerated, without observation of the hypersensitivity or anaphylactic/anaphylactoid reactions that have been seen after infusion of higher doses of nanoparticles. ${ }^{116}$ 
The in vivo antitumor activity of the targeted docetaxelloaded nanoparticles was assessed in mice bearing PSMAexpressing (LNCaP) and PSMA-negative ([breast cancer (MX-1) and nonsmall cell lung cancer (NCI-H460)]) xenograft models, using targeted and nontargeted nanoparticles and free docetaxel for comparison. ${ }^{116}$ For nanoparticles with a long circulation time, an active targeting mechanism improves antitumor efficacy compared with passive targeting nanoparticles in PSMA-positive models. Mechanistically, once nanoparticles enter the tumor tissue, their retention in tumor tissue and specific uptake by cancer cells can be facilitated by active targeting and receptor-mediated endocytosis. This process would result in higher intracellular drug concentrations and increased cytotoxicity. The similar effects of targeted and nontargeted nanoparticles in non-PSMAexpressing tumors are presumably due to increased drug accumulation at tumor sites via the enhanced permeability and retention effect.

Further, the pharmacokinetic profile of the docetaxelloaded nanoparticles (compared with that of the free drug) was investigated in tumor-bearing mice, healthy rats, and nonhuman primates to assess the translation of pharmacokinetic results across different species. The results indicate a high concentration of drug in the plasma compartment, as well as an effective controlled drug release performance. ${ }^{116}$

These preclinical results suggest that nanotherapeutics developed in this way may be beneficial for treatment of solid tumors by improving the therapeutic index of docetaxel through targeting and controlled release of the drug in the tumor microenvironment. Moreover, since the molecular target, PSMA, is expressed on prostate tumor cells and on the neovasculature of other solid tumors, these nanosystems have the potential to increase the utility of docetaxel in the treatment of a range of cancers.

\section{Safety, tolerability, and efficacy of BIND-0 I 4 and range of cancer targets}

The clinical data for BIND-014 are consistent with the observations outlined above. The behavior of BIND-014 in preclinical studies suggests that docetaxel is more effective when formulated as BIND-014 than as Taxotere. More specifically, on the basis of these promising preclinical results, a Phase I clinical trial was undertaken to assess the dose-limiting toxicity and the maximum tolerated dose of BIND-014. ${ }^{116,131}$ The nanochemotherapy was performed by intravenous infusion of BIND-014 to patients with advanced or metastatic cancer, using two dosing schedules, ie, once every 3 weeks (Q3W) and once weekly for 3 weeks followed by one week of no treatment over a 4-week cycle (Q1W). To date, BIND-014 has been clinically tested in over 45 patients with advanced or metastatic cancer who have failed prior therapies.

Based on the widespread expression of PSMA in solid tumor neovasculature, the activity of free docetaxel in many common types of cancer, and the efficacy of BIND-014 in preclinical models of prostate and other cancers, a Phase I study was undertaken to evaluate the efficacy of BIND-014 in a panel of solid tumors. It is worth noting that the pharmacokinetics of docetaxel when released from BIND-014 were markedly similar in humans to those observed in preclinical studies, and that the pharmacokinetics of BIND-014 were different from those of Taxotere in both the preclinical and clinical studies. In the Q3W portion of the study, BIND-014 was well tolerated with predictable and manageable toxicities, and no unexpected toxicity has been observed with BIND014. BIND-014 was demonstrated to be essentially safe and well tolerated, with transient and manageable neutropenia as the dose-limiting toxicity. Other treatment-related adverse events included mild neuropathy, fatigue, mucositis, diarrhea, fluid retention, anemia, rash, hair loss, infusion-related reactions, nail changes, and nausea and vomiting, which are commonly associated with docetaxel as well as many other anticancer drugs. ${ }^{132}$ Further, the maximum tolerated dose was established to be $60 \mathrm{mg} / \mathrm{m}^{2}$ as a 60 -minute infusion under the Q3W treatment regimen. ${ }^{116}$ Using this dose regimen, BIND014 demonstrated antitumor efficacy in nine of 28 patients treated, ranging from one complete response (cervical cancer), three partial responses (non-small-cell lung cancer, metastatic/castration-resistant prostate cancer, and ampullary cancer), and five additional patients with stabilization of disease lasting longer that 12 weeks (pancreatic, colorectal, gallbladder, tonsillar, and anal cancer). These results indicate that the disappearance of cancer evidences in response to treatment, while a partial response refers to a decrease in the size of the tumor or in the extent of cancer in the body.

In summary, the results of a Phase I clinical trial indicate that BIND-014 has a different pharmacologic profile with respect to free docetaxel, including pharmacokinetic properties consistent with a prolonged circulation half-life for BIND-014 and retention of docetaxel in the vascular compartments, with multiple cases of tumor shrinkage at doses up to five times less than the docetaxel dose typically administered. ${ }^{133}$ These results demonstrate that a combination of long nanoparticle circulation time, nanoparticle targeting, and controlled release of docetaxel 
markedly increases the amount of docetaxel delivered to the tumor, and achieves tumor growth inhibition for an extended period of time. Although the Q1W portion of this study is ongoing, Phase II development of BIND-014 as second-line therapy for patients with non-small-cell lung cancer (NCT01792479) ${ }^{134}$ and those with metastatic castrate-resistant prostate cancer (NCT01812746) ${ }^{135}$ was started in 2013 using the Q3W dosing schedule. Further clinical studies to evaluate BIND-014 in a broad range of solid tumors are currently under consideration.

\section{Place in therapy}

Nanotechnologies are having a significant impact on drug delivery, and over recent years, several first-generation therapeutic nanoproducts have been moving ahead towards clinical development. In particular, targeted polymeric nanoparticles, capable of increased cell uptake and enhanced accumulation in target tissue, can be successfully obtained by attaching specific binding entities onto the surface of the nanoparticles. Therefore, active targeting, along with other targeting-based approaches, is envisaged to provide an effective strategy, and several ligand-targeted nanotherapeutics are either approved or under clinical evaluation, leading to second-generation nanomedicines. However, although there are many useful and validated chemical methods for functionalizing nanoparticles with a wide range of targeting ligands, several important issues, including the optimal interplay of physicochemical features, need to be addressed before translation from preclinical to clinical development. Importantly, careful detailing of the physicochemical characteristics of nanoparticles that dictate their in vivo efficacy and a better understanding of the biological and (patho)physiologic principles of drug targeting are need to overcome the current bottlenecks in research and development of rationally designed nanoparticles.

The "drug-targeting" paradigm can be exploited to finally formulate therapeutic carriers that can selectively treat neoplastic tissues without affecting normal cells. The use of targeted nanoparticles in the treatment of cancer, developed in the pioneering work of Farokhzad and Langer, has been validated by the Phase II clinically used prototype BIND-014 (marketed by BIND Therapeutics under the brand name Accurins ${ }^{\mathrm{TM}}$ ), a polymeric drug delivery nanovehicle containing the chemotherapeutic agent docetaxel, which is approved for use in the treatment of several common cancers, including breast, lung, and prostate.

To date, other complex targeted nanosystems addressed to various cancers, also combining diagnostic and therapeutic agents, or that can trigger drug release at the target site when exposed to external stimuli, are currently in clinical development. At this stage, BIND-014 can reasonably be seen as representing an evolution of the "magic bullet" concept, the first description of the drug-targeting paradigm, that can also be used to develop programmable and personalized nanomedicine for routine use in clinic practice.

\section{Acknowledgments}

The authors gratefully acknowledge the Regione Autonoma della Sardegna for financial support grant CRP-25920 "Development of novel targeted nanodevices for the prevention, diagnosis and treatment of prostate cancer", awarded to MS within the frame of "Legge regionale n 7/2007, promozione della ricerca scientifica e dell'innovazione tecnologica in Sardegna, - Annualità 2010”. They also thank Gael McGill (Harvard Medical School) and Digizyme Inc. for their permission to adapt and use the Accurins image.

\section{Disclosure}

The authors report no conflicts of interest in this work.

\section{References}

1. Kim BY, Rutka JT, Chan WC. Nanomedicine. $N$ Engl J Med. 2010;363(25):2434-2443.

2. Peer D, Karp JM, Hong S, Farokhzad OC, Margalit R, Langer R. Nanocarriers as an emerging platform for cancer therapy. Nat Nanotechnol. 2007;2(12):751-760.

3. Zhang L, Gu FX, Chan JM, Wang AZ, Langer RS, Farokhzad OC. Nanoparticles in medicine: therapeutic applications and developments. Clin Pharmacol Ther. 2008;83(5):761-769.

4. Riehemann K, Schneider SW, Luger TA, Godin B, Ferrari M, Fuchs H. Nanomedicine - challenge and perspectives. Angew Chem Int Ed Engl. 2009;48(5):872-897.

5. Doane TL, Burda C. The unique role of nanoparticles in nanomedicine: imaging, drug delivery and therapy. Chem Soc Rev. 2012;41(7):2885-2911.

6. Ferrari M. Cancer nanotechnology: opportunities and challenges. Nat Rev Cancer. 2005;5(3):161-171.

7. Davis ME, Chen ZG, Shin DM. Nanoparticle therapeutics: an emerging treatment modality for cancer. Nat Rev Drug Discov. 2008;7(9):771-782.

8. Farokhzad OC, Langer R. Impact of nanotechnology on drug delivery. ACS Nano. 2009;3(1):16-20.

9. Petros RA, DeSimone JM. Strategies in the design of nanoparticles for therapeutic applications. Nat Rev Drug Discov. 2010;9(8):615-627.

10. Lammers T, Kiessling F, Hennink WE, Storm G. Drug targeting to tumors: principles, pitfalls and (pre-) clinical progress. J Control Release. 2012;161(2):175-187.

11. Jain KK. Role of nanobiotechnology in the development of personalized medicine. Nanomedicine (Lond). 2009;4(3):249-252.

12. Zhang XQ, Xu X, Bertrand N, Pridgen E, Swami A, Farokhzad OC. Interactions of nanomaterials and biological systems: Implications to personalized nanomedicine. Adv Drug Deliv Rev. 2012;64(13): 1363-1384.

13. Gindy ME, Prud'homme RK. Multifunctional nanoparticles for imaging, delivery and targeting in cancer therapy. Expert Opin Drug Deliv. 2009;6(8):865-878. 
14. Janib SM, Moses AS, MacKay JA. Imaging and drug delivery using theranostic nanoparticles. Adv Drug Deliv Rev. 2010;62(11):1052-1063.

15. Lammers T, Kiessling F, Hennink WE, Storm G. Nanotheranostics and image-guided drug delivery: current concepts and future directions. $\mathrm{Mol}$ Pharm. 2010;7(6):1899-1912.

16. Fernandez-Fernandez A, Manchanda R, McGoron AJ. Theranostic applications of nanomaterials in cancer: drug delivery, image-guided therapy, and multifunctional platforms. Appl Biochem Biotechnol. 2011;165(7-8):1628-1651.

17. Yoo D, Lee JH, Shin TH, Cheon J. Theranostic magnetic nanoparticles. Acc Chem Res. 2011;44(10):863-874.

18. Lee DE, Koo H, Sun IC, Ryu JH, Kim K, Kwon IC. Multifunctional nanoparticles for multimodal imaging and theragnosis. Chem Soc Rev. 2012;41(7):2656-2672.

19. Bae KH, Chung HJ, Park TG. Nanomaterials for cancer therapy and imaging. Mol Cells. 2011;31(4):295-302.

20. Taylor A, Wilson KM, Murray P, Fernig DG, Levy R. Long-term tracking of cells using inorganic nanoparticles as contrast agents: are we there yet? Chem Soc Rev. 2012;41(7):2707-2717.

21. Villalonga-Barber C, Micha-Screttas M, Steele BR, Georgopoulos A, Demetzos C. Dendrimers as biopharmaceuticals: synthesis and properties. Curr Top Med Chem. 2008;8(14):1294-1309.

22. Clift MJ, Stone V. Quantum dots: an insight and perspective of their biological interaction and how this relates to their relevance for clinical use. Theranostics. 2012;2(7):668-680.

23. Yamashita T, Yamashita K, Nabeshi H, et al. Carbon nanomaterials: efficacy and safety for nanomedicine. Materials. 2012;5(2):350-363.

24. Partha R, Conyers JL. Biomedical applications of functionalized fullerene-based nanomaterials. Int J Nanomedicine. 2009;4:261-275.

25. Kaur R, Badea I. Nanodiamonds as novel nanomaterials for biomedica applications: drug delivery and imaging systems. Int J Nanomedicine. 2013;8:203-220.

26. Klumpp C, Kostarelos K, Prato M, Bianco A. Functionalized carbon nanotubes as emerging nanovectors for the delivery of therapeutics. Biochim Biophys Acta. 2006;1758(3):404-412.

27. Zhu S, Xu G. Single-walled carbon nanohorns and their applications. Nanoscale. 2010;2(12):2538-2549.

28. Sperling RA, Rivera-Gil P, Zhang F, Zanella M, Parak WJ. Biological applications of gold nanoparticles. Chem Soc Rev. 2008;37(9) 1896-1908.

29. Cai W, Gao T, Hong H, Sun J. Applications of gold nanoparticles in cancer nanotechnology. Nanotechnol Sci Appl. 2008;1:17-32.

30. Lal S, Clare SE, Halas NJ. Nanoshell-enabled photothermal cancer therapy: impending clinical impact. Acc Chem Res. 2008;41(12): 1842-1851.

31. Giljohann DA, Seferos DS, Daniel WL, Massich MD, Patel PC, Mirkin CA. Gold nanoparticles for biology and medicine. Angew Chem Int Ed Engl. 2010;49(19):3280-3294.

32. Dreaden EC, Alkilany AM, Huang X, Murphy CJ, El-Sayed MA. The golden age: gold nanoparticles for biomedicine. Chem Soc Rev. 2012;41(7):2740-2779.

33. Bangham AD, Standish MM, Watkins JC. Diffusion of univalent ions across the lamellae of swollen phospholipids. $\mathrm{J} \mathrm{Mol} \mathrm{Biol}$ 1965;13(1):238-252.

34. Torchilin VP. Recent advances with liposomes as pharmaceutical carriers. Nat Rev Drug Discov. 2005;4(2):145-160.

35. Allen TM, Cullis PR. Drug delivery systems: entering the mainstream. Science. 2004;303(5665):1818-1822.

36. Langer R, Folkman J. Polymers for the sustained release of proteins and other macromolecules. Nature. 1976;263(5580):797-800.

37. Gref R, Minamitake Y, Peracchia MT, Trubetskoy V, Torchilin V, Langer R. Biodegradable long-circulating polymeric nanospheres. Science. 1994;263(5153):1600-1603.

38. Langer R. Drug delivery and targeting. Nature. 1998;392(Suppl 6679): $5-10$.

39. Alexis F, Pridgen EM, Langer R, Farokhzad OC. Nanoparticle technologies for cancer therapy. Handb Exp Pharmacol. 2010;(197):55-86.
40. Shi J, Xiao Z, Kamaly N, Farokhzad OC. Self-assembled targeted nanoparticles: evolution of technologies and bench to bedside translation. Acc Chem Res. 2011;44(10):1123-1134.

41. Walkey CD, Chan WC. Understanding and controlling the interaction of nanomaterials with proteins in a physiological environment. Chem Soc Rev. 2012;41(7):2780-2799.

42. Alexis F, Pridgen E, Molnar LK, Farokhzad OC. Factors affecting the clearance and biodistribution of polymeric nanoparticles. Mol Pharm. 2008;5(4):505-515.

43. Otsuka H, Nagasaki Y, Kataoka K. PEGylated nanoparticles for biological and pharmaceutical applications. Adv Drug Deliv Rev. 2003;55(3):403-419.

44. Avgoustakis K. Pegylated poly(lactide) and poly(lactide-co-glycolide) nanoparticles: preparation, properties and possible applications in drug delivery. Curr Drug Deliv. 2004;1(4):321-333.

45. Betancourt T, Byrne JD, Sunaryo N, et al. PEGylation strategies for active targeting of PLA/PLGA nanoparticles. J Biomed Mater Res A. 2009;91(1):263-276.

46. Jokerst JV, Lobovkina T, Zare RN, Gambhir SS. Nanoparticle PEGylation for imaging and therapy. Nanomedicine (Lond). 2011;6(4): 715-728.

47. Knop K, Hoogenboom R, Fischer D, Schubert US. Poly(ethylene glycol) in drug delivery: pros and cons as well as potential alternatives. Angew Chem Int Ed Engl. 2010;49(36):6288-6308.

48. Hanahan D, Weinberg RA. The hallmarks of cancer. Cell. 2000;100(1):57-70.

49. Hanahan D, Weinberg RA. Hallmarks of cancer: the next generation. Cell. 2011;144(5):646-674.

50. Gabizon A, Shmeeda H, Barenholz Y. Pharmacokinetics of pegylated liposomal doxorubicin: review of animal and human studies. Clin Pharmacokinet. 2003;42(5):419-436.

51. US Food and Drug Administration. Drugs. Available from: http:// www.accessdata.fda.gov/scripts/cder/drugsatfda/index.cfm. Accessed November 5, 2013.

52. Huynh NT, Passirani C, Saulnier P, Benoit JP. Lipid nanocapsules: a new platform for nanomedicine. Int J Pharm. 2009;379(2):201-209.

53. Gradishar WJ, Tjulandin S, Davidson N, et al. Phase III trial of nanoparticle albumin-bound paclitaxel compared with polyethylated castor oil-based paclitaxel in women with breast cancer. J Clin Oncol. 2005;23(31):7794-7803.

54. Montana M, Ducros C, Verhaeghe P, Terme T, Vanelle P, Rathelot P. Albumin-bound paclitaxel: the benefit of this new formulation in the treatment of various cancers. $J$ Chemother. 2011;23(2):59-66.

55. Makadia HK, Siegel SJ. Poly lactic-co-glycolic acid (PLGA) as biodegradable controlled drug delivery carrier. Polymers (Basel). 2011;3(3):1377-1397.

56. Elsabahy M, Wooley KL. Design of polymeric nanoparticles for biomedical delivery applications. Chem Soc Rev. 2012;41(7): 2545-2561.

57. Nicolas J, Mura S, Brambilla D, Mackiewicz N, Couvreur P. Design, functionalization strategies and biomedical applications of targeted biodegradable/biocompatible polymer-based nanocarriers for drug delivery. Chem Soc Rev. 2013;42(3):1147-1235.

58. Kamaly N, Xiao Z, Valencia PM, Radovic-Moreno AF, Farokhzad OC. Targeted polymeric therapeutic nanoparticles: design, development and clinical translation. Chem Soc Rev. 2012;41(7):2971-3010.

59. Strebhardt K, Ullrich A. Paul Ehrlich's magic bullet concept: 100 years of progress. Nat Rev Cancer. 2008;8(6):473-480.

60. Collins I, Workman P. New approaches to molecular cancer therapeutics. Nat Chem Biol. 2006;2(12):689-700.

61. Matsumura Y, Maeda H. A new concept for macromolecular therapeutics in cancer chemotherapy: mechanism of tumoritropic accumulation of proteins and the antitumor agent smancs. Cancer Res. 1986; 46(12 Pt 1):6387-6392.

62. Maeda H, Wu J, Sawa T, Matsumura Y, Hori K. Tumor vascular permeability and the EPR effect in macromolecular therapeutics: a review. J Control Release. 2000;65(1-2):271-284. 
63. Carmeliet P, Jain RK. Angiogenesis in cancer and other diseases. Nature. 2000;407(6801):249-257.

64. Danquah MK, Zhang XA, Mahato RI. Extravasation of polymeric nanomedicines across tumor vasculature. Adv Drug Deliv Rev. 2011;63(8):623-639.

65. Jain RK. Delivery of molecular and cellular medicine to solid tumors. Adv Drug Deliv Rev. 2001;46(1-3):149-168.

66. Jain RK, Stylianopoulos T. Delivering nanomedicine to solid tumors. Nat Rev Clin Oncol. 2010;7(11):653-664.

67. Hatakeyama $\mathrm{H}$, Akita $\mathrm{H}$, Harashima $\mathrm{H}$. A multifunctional envelope type nanodevice (MEND) for gene delivery to tumours based on the EPR effect: a strategy for overcoming the PEG dilemma. Adv Drug Deliv Rev. 2011;63(3):152-160.

68. Valle JW, Armstrong A, Newman C, et al. A phase 2 study of SP1049C, doxorubicin in P-glycoprotein-targeting pluronics, in patients with advanced adenocarcinoma of the esophagus and gastroesophageal junction. Invest New Drugs. 2011;29(5):1029-1037.

69. Matsumura Y, Hamaguchi T, Ura T, et al. Phase I clinical trial and pharmacokinetic evaluation of NK911, a micelle-encapsulated doxorubicin. Br J Cancer. 2004;91(10):1775-1781.

70. Tong R, Cheng J. Anticancer polymeric nanomedicines. Polym Rev (Phila Pa). 2007;47(3):345-381.

71. Singer JW. Paclitaxel poliglumex (XYOTAX, CT-2103): a macromolecular taxane. J Control Release. 2005;109(1-3):120-126.

72. Young C, Schluep T, Hwang J, Eliasof S. CRLX101 (formerly IT-101)-a novel nanopharmaceutical of camptothecin in clinical development. Curr Bioact Compd. 2011;7(1):8-14.

73. Clinical Trials.gov. Study of paclitaxel in patients with ovarian cancer. Available from: http://clinicaltrials.gov/ct2/show/results/ NCT00989131. Accessed November 5, 2013.

74. Tagami T, Ernsting MJ, Li SD. Efficient tumor regression by a single and low dose treatment with a novel and enhanced formulation of thermosensitive liposomal doxorubicin. J Control Release. 2011;152(2):303-309.

75. Seetharamu N, Kim E, Hochster H, Martin F, Muggia F. Phase II study of liposomal cisplatin (SPI-77) in platinum-sensitive recurrences of ovarian cancer. Anticancer Res. 2010;30(2):541-545.

76. Clinical Trials.gov. A Phase I trial of nanoliposomal CPT-11 (NL CPT-11) in patients with recurrent high-grade gliomas. Available from: http:// clinicaltrials.gov/show/NCT00734682. Accessed November 5, 2013.

77. Tardi P, Johnstone S, Harasym N, et al. In vivo maintenance of synergistic cytarabine:daunorubicin ratios greatly enhances therapeutic efficacy. Leuk Res. 2009;33(1):129-139.

78. Byrne JD, Betancourt T, Brannon-Peppas L. Active targeting schemes for nanoparticle systems in cancer therapeutics. Adv Drug Deliv Rev. 2008;60(15):1615-1626.

79. Allen TM. Ligand-targeted therapeutics in anticancer therapy. Nat Rev Cancer. 2002;2(10):750-763.

80. Canton I, Battaglia G. Endocytosis at the nanoscale. Chem Soc Rev. 2012;41(7):2718-2739.

81. Arap W, Pasqualini R, Ruoslahti E. Cancer treatment by targeted drug delivery to tumor vasculature in a mouse model. Science. 1998;279(5349):377-380.

82. Mitra A, Mulholland J, Nan A, McNeill E, Ghandehari H, Line BR. Targeting tumor angiogenic vasculature using polymer-RGD conjugates. $J$ Control Release. 2005;102(1):191-201.

83. Oerlemans C, Bult W, Bos M, Storm G, Nijsen JF, Hennink WE. Polymeric micelles in anticancer therapy: targeting, imaging and triggered release. Pharm Res. 2010;27(12):2569-2589.

84. Deckers R, Moonen CT. Ultrasound triggered, image guided, local drug delivery. J Control Release. 2010;148(1):25-33.

85. Gu F, Langer R, Farokhzad OC. Formulation/preparation of functionalized nanoparticles for in vivo targeted drug delivery. Methods Mol Biol. 2009;544:589-598.

86. Farokhzad OC, Cheng J, Teply BA, et al. Targeted nanoparticleaptamer bioconjugates for cancer chemotherapy in vivo. Proc Natl Acad Sci U SA. 2006;103(16):6315-6320.
87. Gu F, Zhang L, Teply BA, et al. Precise engineering of targeted nanoparticles by using self-assembled biointegrated block copolymers. Proc Natl Acad Sci U S A. 2008;105(7):2586-2591.

88. Yu B, Tai HC, Xue W, Lee LJ, Lee RJ. Receptor-targeted nanocarriers for therapeutic delivery to cancer. Mol Membr Biol. 2010;27(7): 286-298.

89. Schrama D, Reisfeld RA, Becker JC. Antibody targeted drugs as cancer therapeutics. Nat Rev Drug Discov. 2006;5(2):147-159.

90. Chames P, Van Regenmortel M, Weiss E, Baty D. Therapeutic antibodies: successes, limitations and hopes for the future. $\mathrm{Br} J$ Pharmacol. 2009;157(2):220-233.

91. Nobs L, Buchegger F, Gurny R, Allemann E. Biodegradable nanoparticles for direct or two-step tumor immunotargeting. Bioconjug Chem. 2006;17(1):139-145.

92. Weiner LM, Adams GP. New approaches to antibody therapy. Oncogene. 2000;19(53):6144-6151.

93. Ellington AD, Szostak JW. In vitro selection of RNA molecules that bind specific ligands. Nature. 1990;346(6287):818-822.

94. Tuerk C, Gold L. Systematic evolution of ligands by exponential enrichment: RNA ligands to bacteriophage T4 DNA polymerase. Science. 1990;249(4968):505-510.

95. Keefe AD, Pai S, Ellington A. Aptamers as therapeutics. Nat Rev Drug Discov. 2010;9(7):537-550.

96. Xiao Z, Farokhzad OC. Aptamer-functionalized nanoparticles for medical applications: challenges and opportunities. ACS Nano. 2012;6(5):3670-3676.

97. Daniels DA, Chen H, Hicke BJ, Swiderek KM, Gold L. A tenascin-C aptamer identified by tumor cell SELEX: systematic evolution of ligands by exponential enrichment. Proc Natl Acad Sci U S A. 2003;100(26):15416-15421.

98. Farokhzad OC, Jon S, Khademhosseini A, Tran TN, Lavan DA, Langer R. Nanoparticle-aptamer bioconjugates: a new approach for targeting prostate cancer cells. Cancer Res. 2004;64(21):7668-7672.

99. Cheng J, Teply BA, Sherifi I, et al. Formulation of functionalized PLGA-PEG nanoparticles for in vivo targeted drug delivery. Biomaterials. 2007;28(5):869-876.

100. Chang SS, O'Keefe DS, Bacich DJ, Reuter VE, Heston WD, Gaudin PB. Prostate-specific membrane antigen is produced in tumor-associated neovasculature. Clin Cancer Res. 1999;5(10):2674-2681.

101. Ghosh A, Heston WD. Tumor target prostate specific membrane antigen (PSMA) and its regulation in prostate cancer. $J$ Cell Biochem. 2004;91(3):528-539.

102. Schulke N, Varlamova OA, Donovan GP, et al. The homodimer of prostate-specific membrane antigen is a functional target for cancer therapy. Proc Natl Acad Sci U SA. 2003;100(22):12590-12595.

103. Chou LY, Ming K, Chan WC. Strategies for the intracellular delivery of nanoparticles. Chem Soc Rev. 2011;40(1):233-245.

104. Choi CH, Alabi CA, Webster P, Davis ME. Mechanism of active targeting in solid tumors with transferrin-containing gold nanoparticles. Proc Natl Acad Sci U S A. 2010;107(3):1235-1240.

105. Brissette R, Prendergast JK, Goldstein NI. Identification of cancer targets and therapeutics using phage display. Curr Opin Drug Discov Devel. 2006;9(3):363-369.

106. Sugahara KN, Teesalu T, Karmali PP, et al. Coadministration of a tumor-penetrating peptide enhances the efficacy of cancer drugs. Science. 2010;328(5981):1031-1035.

107. Chan JM, Zhang L, Tong R, et al. Spatiotemporal controlled delivery of nanoparticles to injured vasculature. Proc Natl Acad Sci U S A. 2010;107(5):2213-2218.

108. Talekar M, Kendall J, Denny W, Garg S. Targeting of nanoparticles in cancer: drug delivery and diagnostics. Anticancer Drugs. 2011;22(10):949-962.

109. LuY, Low PS. Folate-mediated delivery of macromolecular anticancer therapeutic agents. Adv Drug Deliv Rev. 2002;54(5):675-693.

110. Liang C, Yang Y, Ling Y, Huang Y, Li T, Li X. Improved therapeutic effect of folate-decorated PLGA-PEG nanoparticles for endometrial carcinoma. Bioorg Med Chem. 2011;19(13):4057-4066. 
111. Maresca KP, Hillier SM, Femia FJ, et al. A series of halogenated heterodimeric inhibitors of prostate specific membrane antigen (PSMA) as radiolabeled probes for targeting prostate cancer. $J$ Med Chem. 2009;52(2):347-357.

112. Byun Y, Mease RC, Lupold SE, Pomper MG. Recent development of diagnostic and therapeutic agents targeting glutamate carboxypeptidase II (GCPII). In: Supuran CT, Winum JY, editors. Drug Design of Zinc-Enzyme Inhibitors: Functional, Structural, and Disease Applications. New York, NY: Binghe Wang Wiley Series in Drug Discovery and Development; 2009.

113. Zhou J, Neale JH, Pomper MG, Kozikowski AP. NAAG peptidase inhibitors and their potential for diagnosis and therapy. Nat Rev Drug Discov. 2005;4(12):1015-1026.

114. Sanna V, Pintus G, Roggio AM, et al. Targeted biocompatible nanoparticles for the delivery of (-)-epigallocatechin 3-gallate to prostate cancer cells. J Med Chem. 2011;54(5):1321-1332.

115. Chandran SS, Banerjee SR, Mease RC, Pomper MG, Denmeade SR. Characterization of a targeted nanoparticle functionalized with a urea-based inhibitor of prostate-specific membrane antigen (PSMA). Cancer Biol Ther. 2008;7(6):974-982.

116. Hrkach J, Von Hoff D, Mukkaram Ali M, et al. Preclinical development and clinical translation of a PSMA-targeted docetaxel nanoparticle with a differentiated pharmacological profile. Sci Transl Med. 2012;4(128):128ra139.

117. Wijagkanalan W, Kawakami S, Hashida M. Glycosylated carriers for cell-selective and nuclear delivery of nucleic acids. Front Biosci (Landmark Ed). 2011;16:2970-2987.

118. Hashida M, Nishikawa M, Yamashita F, Takakura Y. Cell-specific delivery of genes with glycosylated carriers. Adv Drug Deliv Rev. 2001;52(3):187-196.

119. Matsumura Y, Gotoh M, Muro K, et al. Phase I and pharmacokinetic study of MCC-465, a doxorubicin (DXR) encapsulated in PEG immunoliposome, in patients with metastatic stomach cancer. Ann Oncol. 2004;15(3):517-525.

120. ClinicalTrials.gov. Safety study of infusion of SGT-53 to treat solid tumors. Available from: http://clinicaltrials.gov/show/ NCT00470613. Accessed November 5, 2013.

121. ClinicalTrials.gov. Study of MBP-426 in patients with second line gastric, gastroesophageal, or esophageal adenocarcinoma. Available from: http://clinicaltrials.gov/show/NCT00964080. Accessed November 5, 2013.

122. Sankhala KK, Mita AC, Adinin R, et al. A phase I pharmacokinetic (PK) study of MBP-426, a novel liposome encapsulated oxaliplatin. J Clin Oncol. 2009;27:S2535.
123. ClinicalTrials.gov. Safety study of CALAA-01 to treat solid tumor cancers. Available from: http://clinicaltrials.gov/show/NCT00689065. Accessed November 5, 2013.

124. Davis ME. The first targeted delivery of siRNA in humans via a selfassembling, cyclodextrin polymer-based nanoparticle: from concept to clinic. Mol Pharm. 2009;6(3):659-668.

125. TrialsUnited. A Phase I study of doxorubicin-loaded anti-EGFR immunoliposomes in patients with advanced solid tumors. Available from: http://www.trialsunited.com/studies/NCT01702129.

126. Mamot C, Ritschard R, Vogel B, et al. A phase I study of doxorubicinloaded anti-EGFR immunoliposomes in patients with advanced solid tumors. J Clin Oncol. 2011;29(15):S3029.

127. Gelmon K. The taxoids: paclitaxel and docetaxel. Lancet. 1994;344(8932):1267-1272.

128. Montero A, Fossella F, Hortobagyi G, Valero V. Docetaxel for treatment of solid tumours: a systematic review of clinical data. Lancet Oncol. 2005;6(4):229-239.

129. Zhang L, Zhang N. How nanotechnology can enhance docetaxel therapy. Int J Nanomedicine. 2013;8:2927-2941.

130. Gref R, Luck M, Quellec P, et al. 'Stealth' corona-core nanoparticles surface modified by polyethylene glycol (PEG): influences of the corona (PEG chain length and surface density) and of the core composition on phagocytic uptake and plasma protein adsorption. Colloids Surf B Biointerfaces. 2000;18(3-4):301-313.

131. ClinicalTrials.gov. A study of BIND-014 given to patients with advanced or metastatic cancer. Available from: http://clinicaltrials. gov/show/NCT01300533. Accessed November 5, 2013.

132. Baker J, Ajani J, Scotte F, et al. Docetaxel-related side effects and their management. Eur J Oncol Nurs. 2009;13(1):49-59.

133. Von Hoff DD, Mita M, Eisenberg P, et al. A Phase 1study of BIND-014, a PSMA-targeted nanoparticle containing docetaxel, in patients with refractory solid tumors. Abstract LB-203 presented at the annual meeting of the American Association for Cancer Research, April 6-10, 2013, Washington, DC, USA.

134. ClinicalTrials.gov. A Phase 2 study to determine the safety and efficacy of BIND-014 (docetaxel nanoparticles injectable suspension) as second line therapy to patients with non-small cell lung cancer. Available from: http://clinicaltrials.gov/show/NCT01792479. Accessed November 5, 2013

135. ClinicalTrials.gov. A Phase 2 study to determine the safety and efficacy of BIND-014 (docetaxel nanoparticles injectable suspension), administered to patients with metastatic castration-resistant prostate cancer. Available from: http://clinicaltrials.gov/show/NCT01812746. Accessed November 5, 2013.
International Journal of Nanomedicine

\section{Publish your work in this journal}

The International Journal of Nanomedicine is an international, peerreviewed journal focusing on the application of nanotechnology in diagnostics, therapeutics, and drug delivery systems throughout the biomedical field. This journal is indexed on PubMed Central,

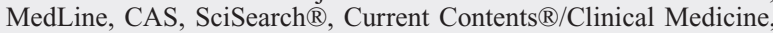

\section{Dovepress}

Journal Citation Reports/Science Edition, EMBase, Scopus and the Elsevier Bibliographic databases. The manuscript management system is completely online and includes a very quick and fair peer-review system, which is all easy to use. Visit http://www.dovepress.com/ testimonials.php to read real quotes from published authors. 